\title{
The flash crash: a review
}

\author{
Ali N. Akansu \\ Department of Electrical and Computer Engineering, \\ New Jersey Institute of Technology, Newark, New Jersey, USA
}

\begin{abstract}
Purpose - The purpose of this paper is to present an overview of the flash crash, and explain why and how it happened.

Design/methodology/approach - The author summarizes several studies suggesting various perspectives on the flash crash and its causes. Furthermore, the author highlights recently proposed and introduced improvements and regulations to reduce the risk of having similar market collapses in the future. Findings - It is an overview paper that highlights the state of the art on the subject.

Research limitations/implications - Paper does not report any research findings of the author.

Practical implications - High-frequency trading (HFT) along with its pros and cons is the new normal for most of the current electronic trading activity in the markets. It is well recognized by the experts that HFT may have its important shortcomings whenever the rules and regulations are not up to date to match the technological progress offering faster computational and execution capabilities.

Social implications - HFT has created a societal discussion about its benefits and potential deficiencies as the common practice for trading due to potentially unequal access to market data by various categories of participants. Such arguments help the regulators to develop improvements to reduce the market risk and nurture more robust and fair markets for all.

Originality/value - The paper has a tutorial value and summarizes the current state of HFT. The readers of more interest are guided to the most relevant literature for further reading.

Keywords Algorithmic trading, Electronic trading, High-frequency trading (HFT), Limit order book (LOB), The flash crash, LOB imbalance, Security Information Processor (SIP), National best bid and offer (NBBO), Regulation national market system (Reg NMS), United States Securities and Exchange Commission (SEC)

Paper type Research paper
\end{abstract}

\section{Introduction}

US equity markets with about 8,000 tickers literally collapsed within a few minutes and quickly recovered by the end of the day on May 6, 2010. They were down in the range of $5-15$ percent, and a few hundred of them lost 60 percent of their values in this short period of time. The markets recovered most of their losses at the end of the day, and closed the day 3 percent down from the close of the previous day. The market has lost about $\$ 1$ trillion of its value during this episode due to the chaotic nature of volatility and disappearance of liquidity that made limit order books (LOBs) of highly correlated equities extremely unstable and dysfunctional, and the entire system collapsed. This event, called "the Flash Crash", was the first of its kind experienced by the financial industry and generated serious concerns, and highlighted the weaknesses of financial markets and some of the regulations (SEC, 2010a, b; Lewis, 2015). Mostly, high-frequency trading (HFT) has been presented as the culprit by many experts and writers that created a societal discussion about the use of the-state-of-the-art technology in the financial sector. The jury is still out although HFT has become the new normal for the trading infrastructure in the USA.

In this paper, we present an overview of the flash crash, and explain why and how it happened. We summarize several studies suggesting various perspectives on the flash crash and its causes (SEC, 2010a, b; Bouchaud et al., 2002). Furthermore, we highlight

(C) Ali N. Akansu. Published in Journal of Capital Markets Studies. Published by Emerald Publishing Limited. This article is published under the Creative Commons Attribution (CC BY 4.0) licence. Anyone may reproduce, distribute, translate and create derivative works of this article (for both commercial \& non-commercial purposes), subject to full attribution to the original publication and authors. The full terms of this licence may be seen at http://creativecommons.org/licenses/by/4.0/legalcode

Received 2 July 2017 Revised 18 July 2017 Accepted 4 August 2017 
JCMS

1,1 recently proposed and introduced improvements and regulations to reduce the risk of having similar market collapses in the future.

The price formation process of an asset in an exchange and its consolidated version for the multiple exchanges based trading infrastructure are discussed in the following section. In Section 3, HFT is presented and its unique characteristics are highlighted. Then, we detail the underlying events of May 6, 2010 and dramatic regime change in the financial markets that triggered the collapse in Section 4. The experiences gained and lessons learned from the flash crash are summarized in Section 5. Then, we conclude the paper.

\section{LOB and price formation of an asset}

LOB is the list of ask and bid prices along with attached quantities and order types of a tradable financial asset. The information posted on an LOB is continuously checked and updated by the computationally powerful matching engine of an electronic trading platform that executes and reports trades of a single ticker in real time at an exchange. Commonly, a single ticker is simultaneously traded at multiple exchanges. In the USA, these exchanges periodically report their best quotes (bids and offers) and executed trades to the Security Information Processor (SIP) (Consolidated Tape Association (CTA)) that functions as the benchmark of the market. These data are consolidated and displayed in real time as the National Best Bid and Offer (NBBO) governed by the Regulation National Market System (Reg NMS) that is described and enforced by the United States Securities and Exchange Commission (SEC) (Consolidated Tape Association Plan/Consolidated Quotation Plan (CTA/CQ); Regulation National Market System (Reg NMS), 2005). Regulation NMS sets the rules to ensure that trades occur at the best available prices at any time in order to achieve a robust, transparent and fair market for all participants.

Mathematically speaking, the price formation process of a stock through the market microstructure as highlighted is sophisticated with its intricacies. In theory, the price of a stock is usually modeled as the geometric Brownian motion in which the rate of return (price change) for a stock is defined as Bachelier (1900), and Akansu and Torun (2017):

$$
\frac{d p(t)}{p(t)}=\mu t+\sigma w(t), \quad t \geqslant 0
$$

where $p(t)$ is the price of the stock, $\mu$ the drift, $\sigma$ the volatility or the standard deviation of price change (return) and $w(t)$ the standard Brownian motion. This stochastic differential equation has its solution obtained by using Ito's Lemma and expressed as Ito (1946):

$$
p(t)=p(0) \exp \left[\left(\mu-\frac{\sigma^{2}}{2}\right) t+\sigma w(t)\right]
$$

It is noted that geometric Brownian motion model assumes constant volatility. Since prices of stocks are affected by economic news and many relevant events that happen almost randomly with their unique impact, constant volatility assumption may not always hold. The flash crash is the case in point thoroughly investigated in the paper. More advanced price models incorporate the very fact that the volatility itself varies in time, and they include time local volatility in the formulation. Therefore, more realistic model is shown as:

$$
\frac{d p(t)}{p(t)}=\mu t+\sigma[p(t), t] w(t), \quad t \geqslant 0
$$

where $\sigma[p(t), t]$ is the volatility at time $t$. 
Discrete-time counterpart of geometric Brownian motion model is obtained by sampling the price and given as (Akansu and Torun, 2017):

$$
s(n)=s(n-1)+\mu+\sigma \xi(n)
$$

where $s(n)=\operatorname{In} p(n)$ is the log-price of a stock at discrete-time $n$ with price $p(n), \mu$ the drift, $\sigma$ is volatility of the stock, and, $\xi(n)$ the white Gaussian with zero mean and unit variance, $\xi(n) \sim \mathcal{N}(0,1)$.

Limit orders sent by the market participants with their price, volume and trade limiting requirements are aggregated by the price and time priority (PTP) protocol and posted in the LOB. These limit orders are matched with market orders of the same price by the matching engine in the order execution process that creates trades. Trading cycle is completed through the settlement steps and recorded permanently as a trade at the exchange (Hasbrouck, 2007). The order executions to buy and sell assets causes price fluctuations that are formulated as the market impact (Cont, 2011). Execution strategies are developed to lower market impact at favorable prices and times identified through the real-time analysis of LOB structure and its variations (Akansu and Torun, 2017).

With the help of the example given in Akansu and Torun (2017), we explain herein the inner-workings of the LOB step by step for the limit and market order types, and track how they interact and impact the book to create a dynamically changing market for an asset in an exchange. Four LOB snapshots for a ticker are shown in Figure 1 to describe the LOB states just before and right after a limit order to buy 100 shares of let's say a stock at $\$ 98.75$ per share is placed by a trader. There are five limit orders at four different price levels prior to this order as seen in Figure 1(a). The lowest priced two limit orders are on the buy side (bid) while the remaining three orders are placed at the two highest price levels of the sell side (ask) where $\$ 98.77$ price range has two independent orders sent to the LOB. The bid-ask spread (spread) of the LOB at this time and state is $\$ 0.01$. Then, 100 shares of limit order to buy at $\$ 98.75$ arrive and placed in the book based on the PTP protocol as depicted in Figure 1(b). Later in time, a market order of 200 shares to sell at $\$ 98.75$ arrives at the LOB, and it is matched with the first arrived limit order of 200 shares sitting in the front of the best bid level, $\$ 98.75$. Therefore, the available shares at that price level of the LOB are depleted by 200 as displayed in Figure 1(c). Next, the trader who placed the limit order to buy 100 shares at $\$ 98.75$ cancels its order as depicted in Figure 1(d). It is noted that the bid-ask spread of the LOB at this time becomes $\$ 0.02$. Similar to this example, such trading activities in an exchange create the ask (supply) and bid (demand) sides of the market for an asset through its LOB at that exchange. Typically, an asset is simultaneously traded at multiple exchanges with their own LOBs for the ticker. The status of these LOBs for the same ticker are periodically reported to the centralized SIP (Consolidated Tape Association (CTA)) by all the exchanges in order to post the NBBO that is utilized as the market reference by the participants and regulators (Consolidated Tape Association Plan/Consolidated Quotation Plan (CTA/CQ); Reg NMS, 2005). The trading dynamics of LOBs and the stability of the resulting NBBO lead to the market price formation and volatility (risk) of the asset under consideration where its market maker(s), with their privileges and responsibilities, play(s) a crucial role. The number of price levels and the number of orders (with quantities) in each level (complete book) are quite larger (in hundreds for some liquid stocks) in a real-world scenario than what we have in this example. The price level (bin) resolution of $\mathrm{LOB}$ and available order types may vary based on asset type and trading rules set by regulatory bodies, financial and legal frameworks that are implemented and serviced by exchanges.

It is desirable to describe the inner workings of LOB through the models where other significant metrics like the probability of upward/downward price moves (trends) based on the current and most recent LOB states can be analytically expressed (Cont et al., 2010). 
JCMS

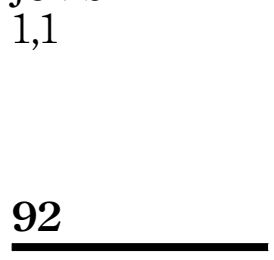

Figure 1.

An example to show changes in LOB as time goes (a)

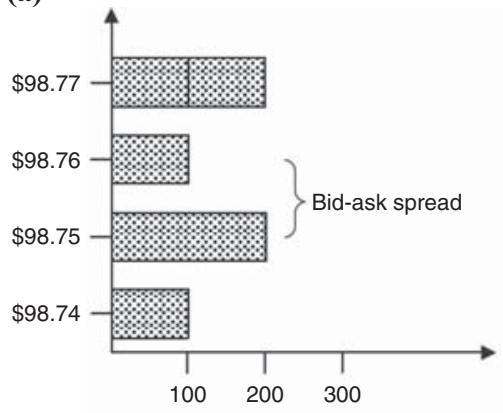

(c)

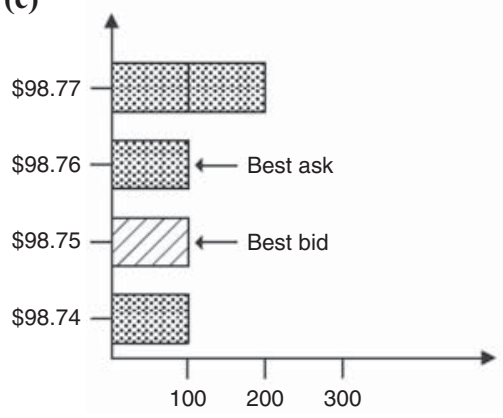

(b)

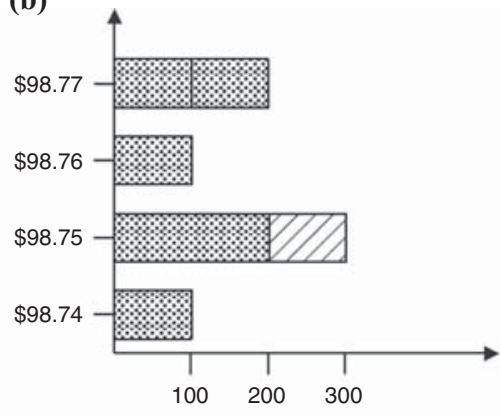

(d)

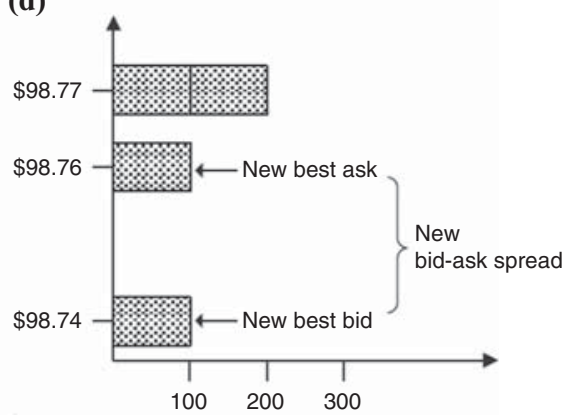

Notes: (a) There are five limit orders at four different price levels at the initial state. The bid-ask spread is $\$ 0.01$; (b) the limit order to buy 100 shares at $\$ 98.75$ arrives and placed according to PTP protocol; (c) the market order to sell 200 shares at $\$ 98.75$ arrives and matched with the first arrived limit order sitting at the best bid; (d) the bidding limit order placed in (b) is canceled. New best bid is $\$ 98.74$ and the new bid-ask spread is $\$ 0.02$ (Akansu and Torun, 2017)

Note that a thorough understanding of LOB along with the technological and procedural specifics of the trading venue always help us to have better insight and intuition to build more complex proprietary HFT strategies (Akansu and Torun, 2017).

Interested readers are referred to Hasbrouck (2007), Bouchaud et al. (2002), Potters and Bouchaud (2003), Jain et al. (2016), and Menkveld (2016) for further discussions on LOBs, and market microstructure in general. The applications of LOB models in optimal order execution are covered in Alfonsi et al. (2010), Alfonsi and Schied (2010), Bayraktar and Ludkovski (2014). Moreover, references (Avellaneda and Stoikov, 2008; Guilbaud and Pham, 2013) present applications in HFT. Furthermore, an overview on modeling of high-frequency financial data is found in Cont (2011).

\section{HFT}

The advances in technology and its deployment in financial sector have caused a historical disruption in several aspects of the financial activity. Warren Buffet remarked on HFT as "one of the most significant market structure developments in recent years (SEC, 2010a, b; Crippen, 2014)." This paradigm shift made leading firms of old models fall behind, losing capacity and market share as new models evolve and flourish. This "new normal" with its fierce competition and technology driven nature along with the unpreparedness of the 
regulatory bodies to upgrade the rules and regulations, and fueled by various interest groups generated public outcry and a negative social reaction against HFT for the last few years (Lewis, 2015). The pros and cons of HFT has been extensively debated by many people arguing different aspects of such a complex issue including Buffet who stated his view on HFT as "It's not a liquidity provider. It may create more volume but that's not the same as being a liquidity provider. To the extent that it is front running, I think society has generally been against front running for good reasons. Here they've gained an advantage by figuring out how the system worked and getting there first and that adds nothing to economic activity. On the other hand, high-frequency trading isn't costing the average investor a penny. They've never had it so good" (Crippen, 2014). Today, most of the tradings in the financial markets are HFT, and it is a quite matured field with its evolving business models since the speed is a commodity now (Parsons, 2017).

Technology Advisor Committee's working group of the US Commodity Futures Trading Commission (CFTC) identified the following features of HFT as its unique descriptors (CFTC, 2013):

(1) Algorithms for decision making, order initiation, generation, routing or execution, for each individual transaction without human direction.

(2) Low-latency technology that is designed to minimize response times, including proximity and co-location services.

(3) High-speed connections to markets for order entry.

(4) Recurring high message rates (orders, quotes or cancellations) determined using one or more objective forms of measurement, including cancel-to-fill ratios; participantto-market message ratios; or participant-to-market trade volume ratios. And, effectively, HFT is a form of automated trading, but not all automated trading is HFT.

According to the SEC, the main characteristics of HFT firms include:

(1) their use of extraordinarily high-speed and sophisticated computer programs for generating, routing and executing orders;

(2) their use of co-location services and individual data feeds offered by exchanges and others to minimize network and other types of latencies;

(3) their use of very short time-frames for establishing and liquidating positions;

(4) their submission of numerous orders that are canceled shortly after submission; and

(5) their ending the trading day in as close to a flat position as possible (i.e. not carrying significant, unhedged positions over-night) (SEC, 2010a, b).

A typical HFT trade account satisfies the three metrics: trading more than 5,000 contracts per day (a notional value of more than $\$ 300$ million in August 2010); holding end of day inventory positions of no more than 5 percent of its total daily volume; and maintaining intraday inventory positions of less than 10 percent of its total daily volume (Baron et al., 2012).

The literature is rich in studies on market microstructure and HFT. The SEC staff published excellent white papers reviewing these publications. Part I entitled "Market Fragmentation" covers reviews of papers published between 2007 and 2013 that address the issue of market fragmentation - both visible and dark by analyzing financial market data and reach empirical findings (US Securities and Exchange Commission, 2013). Similarly, Part II is entitled "HFT" and discusses papers that address the particular issues of HFT. These papers analyze non-public data sets where market activity can be attributed to trading accounts that have been identified as engaging in HFT (US Securities and Exchange Commission, 2014). HFT is a large subset, but by no means all, of algorithmic trading. 
JCMS

1,1

94

For example, algorithmic trading describes a broad range of trading activity, also including the execution algorithms of large orders. This type of algorithm takes large size orders with the potential of causing high market impact and breaks them into many small size orders that are spread in time for their execution. Such smart execution algorithms to liquidate large positions through many small size high-frequency trades with the overall longer time horizons for order completion are not classified as HFT in this white paper.

Although HFT encompasses a variety of strategies, we briefly discuss a few of these methods here and the readers of more interest are referred to the references for further reading (Akansu and Torun, 2017; US Securities and Exchange Commission, 2014). Market making is one of the most common HFT strategies. It is based on submission of non-marketable orders providing liquidity at pre-defined prices, and at both ask and bid sides of the LOB. This strategy profits from the spread between bids and offers along with rebates paid by exchanges as the incentive to provide liquidity. It is noted that a traditional market maker is required to provide liquidity while an HFT market maker does not have any obligation to provide liquidity for the market. The optimal pricing algorithms of market makers consider the built in asset price risk and information risk due to informed traders in the market. The statistical properties of LOB are tightly coupled in the development of such market making strategies. A variety of HFT arbitrage strategies are utilized by traders. An arbitrage strategy seeks to capture price inefficiencies between related and correlated products, venues, and markets. For example, one may arbitrage pricing of an exchange traded fund (ETF) against its underlying basket of stocks with the assumption that market efficiency causes mean reverting asset returns. Structural strategies attempt to exploit the built-in inefficiencies in the market, in practices of certain market participants or in certain products. As an example, traders with access to the low-latency market data and powerful processing tools may profit by low-latency (speed) trading with market participants using a venue offering executions at stale prices. Similarly, directional strategies have their variations including momentum ignition and order anticipation types. These directional HFT strategies have been a major concern with their potential to disturb the natural price formation process through their manipulative and predatory versions. For example, a momentum ignition strategy may initiate a sequence of orchestrated orders and trades in order to cause a rapid price change up or down to benefit from. This kind of manipulative (predatory) trading strategy is an illegal practice with its consequences (Akansu and Torun, 2017; US Securities and Exchange Commission, 2014). Indeed, there are claims among others that such a trading pattern caused the flash crash of May 6, 2010 that discussed in the next section.

\section{The flash crash}

On May 6, 2010, trading day started with political and economic news from overseas regarding the European debt crisis. Therefore, risk premiums rose to protect against likely default by the Greek government on their sovereign debt. The Euro began its sharp decline around 1:00 p.m. against US Dollar and Japanese Yen (SEC, 2010a, b).

Report of the staffs of the CFTC and SEC to the Joint Advisory Committee on Emerging Regulatory Issues provided an in-depth analysis of the flash crash of May 6, 2010 (SEC, $2010 \mathrm{a}, \mathrm{b})$. We summarize herein the findings of the report and significant events that triggered this historical market collapse as follows:

(1) Around 1:00 p.m., broadly negative market sentiment was already affecting an increase in the price volatility of some individual securities. And, the number of volatility pauses, Liquidity Replenishment Points, triggered on the New York Stock Exchange in individual equities began to substantially increase above their average levels (SEC, 2010a, b). 
(2) By 2:30 p.m., the S\&P 500 volatility index was up 22.5 percent from the opening level, yields of ten-year treasuries fell and selling pressure had pushed the Dow Jones Industrial Average down about 2.5 percent. Buy-side liquidity in the E-Mini S\&P 500 futures contracts (the "E-Mini"), the S\&P 500 ETF ("SPY"), the two most active stock index instruments traded in equity markets, had fallen 55 and 20 percent, respectively. Some individual stocks also suffered from a decline in their liquidity (SEC, 2010a, b).

(3) At 2:32 p.m., in addition to already unusually high volatility and thinning liquidity, a large fundamental trader initiated a sell program to sell a total of 75,000 E-Mini contracts (valued at approximately $\$ 4.1$ billion) as a hedge to an existing equity position.

(4) This large fundamental trader chose to execute this sell program via an automated execution algorithm. It was programmed to feed orders into the June 2010 E-Mini market to target an execution rate set to 9 percent of the trading volume calculated over the previous minute without checking market price or time:

- It is expected from such an execution algorithm for large size sell program to take into account of price, time and volume information at the current market in order to keep its market impact within a justifiable range.

- The execution of this sell program was extremely fast, in just 20 minutes compared to five hours for a prior execution of the same size sell order (SEC, $2010 \mathrm{a}, \mathrm{b})$, and resulted in the largest net change in daily position of any trader in the E-Mini since the beginning of the year in 2010.

This strong sell pressure was initially absorbed by:

- high-frequency traders ("HFTs") and other intermediaries in the futures market;

- fundamental buyers in the futures market; and

- cross-market arbitrageurs who transferred this sell pressure to the equities markets through their opportunistic buy of E-Mini contracts and simultaneously selling highly correlated products like SPY, or selling individual equities in the S\&P 500 Index (SEC, 2010a, b).

What happened next is best described in terms of the two strongly coupled liquidity crises. Namely, first one is at the broad index level in the E-Mini, and the second one is with respect to individual stocks as described in the following:

(1) Liquidity crisis in the E-Mini (SEC, 2010a, b):

- Between 2:32 p.m. and 2:45 p.m., as prices of the E-Mini rapidly declined, the sell algorithm sold about 35,000 E-Mini contracts of the 75,000 intended. During the same time, all fundamental sellers combined sold more than 80,000 contracts net, while all fundamental buyers bought only about 50,000 contracts net, for the resulting net fundamental imbalance of 30,000 contracts. This level of net selling by fundamental sellers is about 15 times larger compared to the same 13-minute interval during the previous three days, while this level of net buying by the fundamental buyers is about ten times larger compared to the same time period during the previous three days.

- At 2:45:28 p.m., trading on the E-Mini was paused for "five seconds" when the Chicago Mercantile Exchange circuit breaker was triggered in order to prevent a cascade of further price declines. In that short period of time, sell-side pressure in the E-Mini was partly alleviated and buy-side interest increased. When trading 
JCMS

1,1

96

resumed at 2:45:33 p.m., prices stabilized and shortly thereafter, the E-Mini began to recover, followed by the SPY. The sell algorithm continued to execute the sell program until about 2:51 p.m. as the prices were rapidly rising in both the E-Mini and SPY:

(2) Liquidity crisis with respect to individual stocks (SEC, 2010a, b):

- The second liquidity crisis occurred in the equities markets at about 2:45 p.m. Automated trading systems used by many liquidity providers temporarily paused in reaction to the sudden price declines observed during the first liquidity crisis (SEC, 2010a, b).

- After their trading systems were automatically paused, they had to assess the risks associated with continuing their trading.

- By approximately 3:00 p.m., most securities had reverted back to trading at prices reflecting true consensus values (SEC, 2010a, b).

- During the 20-minute period between 2:40 p.m. and 3:00 p.m., over 20,000 trades (many retail-customer orders) across more than 300 separate securities were executed at prices 60 percent or more away from their 2:40 p.m. prices (SEC, 2010a, b).

Table I tabulates summary of statistics for the June 2010 E-mini S\&P 500 futures contract for May 3-5 and May 6, 2010 (Kirilenko et al., 2017). Similarly, Figure 2 presents minute-byminute transaction prices and trading volume of the June 2010 E-mini S\&P futures contract on May 6, 2010 between 8:30 and 15:15 CT. Trading volume is calculated as the number of contracts traded during each minute. Transaction price is the last transaction price of each minute.

\section{Lessons learned}

The undesirable events with their underlying causes that triggered the market disorientation and eventually its collapse on May 6, 2010 were explained earlier in the paper. And in this section, we highlight lessons learned by all stakeholders including financial sector, governmental and non-governmental regulatory bodies and the public. We provide the summary of a potpourri of proposals made by several institutions and researchers to improve market stability and robustness in order to avoid similar crashes in the future. These developments have created public debate on HFT, and relevant laws and regulations are duly revised, accordingly, by legislative and regulatory bodies. Moreover, a clear understanding of the flash crash has generated significant know-how, and the leading regulators and exchanges around the globe have benefited from this experience to improve their operations and regulations for HFT practices.

\begin{tabular}{lcc}
\hline & May 3-5 & May 6 \\
\hline Daily trading volume & $2,397,639$ & $5,094,703$ \\
No. of trades & 446,340 & $1,030,204$ \\
No. of traders & 11,875 & 15,422 \\
Trade size & 5.41 & 4.99 \\
Limit orders \% volume & 95.45 & 92.44 \\
Limit orders \% trades & 94.36 & 91.75 \\
Volatility (log high-low price range) (\%) & 1.54 & 9.82 \\
Return (\%) & -0.02 & -3.05 \\
Source: Kirilenko et al. $(2017)$ & & \\
\end{tabular}

Table I.

Statistics for the June 2010 E-mini S\&P 500 futures contract for May 3-5 and May 6, 2010

\footnotetext{
Source: Kirilenko et al. (2017)
} 


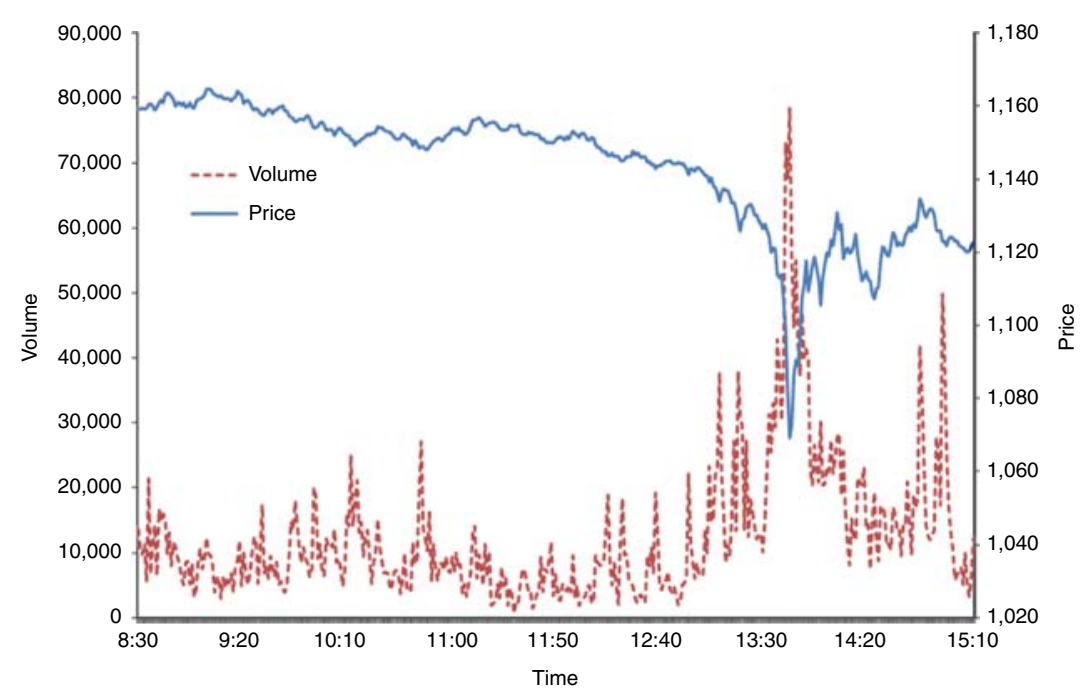

Source: SEC (2010a, b)

We summarize the major observations and conclusions drawn from the chaotic market conditions of May 6, 2010 as follows:

(1) The smart execution of a large size sell order can trigger extreme price movements under stressed market conditions with high volatility. The co-existence of smart execution and algorithmic trading in a typical exchange can quickly deplete liquidity and result in unstable LOB and executed trades that are not economically meaningful.

(2) The flash crash was also an eye opener to remind us the integrated nature of derivatives and securities markets. The cross-market trading activity detailed above, in particular, the price discovery cross-products mimicking an index like the E-Mini and SPY was confirmed and verified by extensive investigations and research. The Joint Advisory Committee on Emerging Regulatory Issues was formed prior to the flash Crash due to the need for coordinated regulatory solution for the well observed convergence between securities and derivatives markets including the better monitoring and more judicious recalibration of the existing market-wide circuit breakers that did not turn on even on May 6, 2010.

(3) Commonly, each market participant has its own trade stoppers (pauses) to withdraw from trading of a specific ticker, product, basket or other category based on the relevant measurements and automated decisions. Mostly, these withdrawals happen almost randomly and sporadically and do not impact the entire market. Whenever many withdrawals from trading of a single ticker occur, naturally, its LOB is stressed and its spread becomes economically unjustified. Moreover, if such a ticker is highly coupled with a security heavily used by many participants in the market to hedge open positions against market risk, e.g. E-Mini with SPY, then the LOB of the ticker becomes highly stressed and volatile. Hence, the market stoppers need to be devised based on the specifics and roles of tickers in the markets. "One fits for all" approach is proven to collapse LOBs and markets. Therefore, the SEC worked with the exchanges and FINRA to implement a circuit breaker to maintain LOB of each security. Such a circuit breaker stops trading across the US markets in a security for 
JCMS

1,1

98 five minutes if its price has changed 10 percent during the last five minutes. The SEC approved the use of these circuit breakers to securities that belong to the S\&P 500 and Russell 1000 indices and certain ETFs.

(4) The financial commitment of a market participant in a specific ticker is unknown. Similarly, its decision protocols to participate or to withdraw from the trading of a ticker is proprietary. In a stressed and volatile market environment, these uncertainties increase along with the elevated uncertainty of when the ticker base market stopper will kick in to pause trading. The latter systematically impacts the first two sets of decision thresholds emphasized here that impact the available liquidity in the LOB. It is reported that the exchanges and FINRA only broke trades that were more than 60 percent away from the applicable reference price through a process that was not transparent. In order to fill the void, the SEC approved the new trade break procedures similar to the new circuit breakers.

The observations made and the experiences gained by the participants and stakeholders due to the out of the ordinary events and outcomes of the flash crash have motivated various players to come up with their proposals to address the weaknesses in the market structure and regulations for HFT. An incomplete list of the proposed procedures to improve HFT practices by various experts includes order cancellation fees, minimum order exposure times, transaction tax, requiring algorithmic trading firms to register with FINRA as discussed in Vazquez (2017), Dolgopolov (2017), McPartland (2013) and their references. In McPartland (2013), the author discusses the advantages of HFT along with the shortcomings of the current regulations where questionable HFT practices may still happen since the regulatory enforcement in the granular level of trading algorithms is almost impossible. He made nine specific recommendations for improved market microstructure to support more fair HFT spanning from having a unique identifier for each automated trading system (each individual algorithm) to be used for the audit trails to the strict prevention of receiving any trade information directly from the trade matching engines prior to its availability to all market participants.

\section{Conclusions}

The HFT infrastructure of the markets in the USA has been disruptive with its built-in efficiencies for the financial sector. And, it is commonly agreed that it is also good for the public when judiciously regulated. This rapid transformation has created its own challenges. The traders, portfolio managers and investment firms along with regulators and investors were caught unprepared and inexperienced for such a paradigm shift. Although prior mini-crashes were experienced in the markets, the scale and impact of the flash crash made it historical. In this paper, we presented an overview of the flash crash and its causes drawn from in-depth investigations and studies reported by experts and researchers. The educative value of this experience is significant, and the regulations for HFT have been continuously revisited and being upgraded by the responsible bodies. Although the mechanics and the events triggered the flash crash are well understood by the experts in HFT, there are still a number of loopholes, questionable practices and business models of the exchanges and traders that need to be streamlined and addressed sooner than later.

\section{References}

Akansu, A.N. and Torun, M.U. (2017), A Primer for Financial Engineering: Financial Signal Processing and Electronic Trading, Elsevier, London.

Alfonsi, A. and Schied, A. (2010), "Optimal trade execution and absence of price manipulations in limit order book models”, SIAM Journal of Financial Mathematics, Vol. 1 No. 1, pp. 490-522. 
Alfonsi, A., Fruth, A. and Schied, A. (2010), "Optimal execution strategies in limit order books with general shape functions", Quantitative Finance, Vol. 10 No. 2, pp. 143-157.

Avellaneda, M. and Stoikov, S. (2008), "High-frequency trading in a limit order book", Quantitative Finance, Vol. 8 No. 3, pp. 217-224.

Bachelier, L. (1900), "Théorie de la spéculation”, Annales scientifiques de l'École Normale Supérieure, Vol. 3, pp. 21-86, available at: www.numdam.org/item?id=ASENS_1900_3_17_21_0

Baron, M., Brogaard, J. and Kirilenko, A. (2012), "The trading profits of high frequency traders", available at: https://faculty.chicagobooth.edu/john.cochrane/teaching/35150_advanced_ investments/Baron_Brogaard_Kirilenko.pdf (accessed January 2013).

Bayraktar, E. and Ludkovski, M. (2014), "Liquidation in limit order books with controlled intensity", Mathematical Finance, Vol. 24 No. 4, pp. 627-650.

Bouchaud, J.P., Mezard, M. and Potters, M. (2002), "Statistical properties of stock order books: empirical results and models", Quantitative Finance, Vol. 2 No. 4, pp. 251-256.

Brogaard, J.A. (2010), "High frequency trading and its impact on market quality", September.

CFTC (2013), "Concept release on risk controls and system safeguards for automated trading", Commodity Futures Trading Commission (CFTC), RIN 3038-AD52, September 2013, available at: www.cftc.gov/idc/groups/public/@newsroom/documents/file/federalregister090913.pdf

Consolidated Tape Association (CTA), "Facts about the security information processor (SIP)", available at: www.ctaplan.com/index

Consolidated Tape Association Plan/Consolidated Quotation Plan (CTA/CQ), available at: www.sec. gov/rules/sro/nms.htm\#ctacq

Cont, R. (2011), "Statistical modeling of high-frequency financial data", IEEE Signal Processing Magazine, Vol. 28 No. 5, pp. 16-25.

Cont, R., Stoikov, S., Talreja, R. and Stochastic, A. (2010), "Model for order book dynamics", Operations Research, Vol. 58 No. 3, pp. 549-563.

Crippen, A. (2014), "Buffett, Gates and Munger criticize high-frequency Trading”, CNBC (May 5, 2014), available at: www.cnbc.com/2014/05/05/buffett-gates-and-munger-criticize-high-frequencytrading.html

Dolgopolov, S. (2017), "Securities fraud embedded in the market structure crisis: high-frequency traders as primary violators", William \& Mary Business Law Review, (Forthcoming), available at: https:// papers.ssrn.com/sol3/papers.cfm?abstract_id=2980136

Guilbaud, F. and Pham, H. (2013), "Optimal high-frequency trading with limit and market orders", Quantitative Finance, Vol. 13 No. 1, pp. 79-94.

Hasbrouck, J. (2007), Empirical Market Microstructure: The Institutions, Economics, and Econometrics of Securities Trading, Oxford University Press, New York, NY.

Ito, K. (1946), "On a stochastic integral equation", Proceedings of Japan Academy, Vol. 22 Nos 1-4, pp. 32-35.

Jain, P.K., Jain, P. and McInish, T.H. (2016), “Does high-frequency trading increase systemic risk?”, Journal of Financial Markets, Vol. 31, pp. 1-24.

Kirilenko, A., Kyle, A.S., Samadi, M. and Tuzun, T. (2017), “The flash crash: high-frequency trading in an electronic market”, Journal of Finance, (Forthcoming), available at: https://papers.ssrn.com/ sol3/papers.cfm?abstract_id-1686004

Lewis, M. (2015), Flash Boys: A Wall Street Revolt, 1st ed., W.W. Norton \& Company, New York, NY.

McPartland, J. (2013), "Recommendations for equitable allocation of trades in high frequency trading environments", Policy Discussion Paper, Vol. PDP, No. 2013-01, Chicago, IL, May, available at: www.chicagofed.org/publications/policy-discussion-papers/2013/pdp-1 (accessed July 2014).

Menkveld, A.J. (2016), "The economics of high-frequency trading: taking stock", Annual Review of Financial Economics, Vol. 8 No. 1, pp. 1-24. 
JCMS

1,1
Parsons, J. (2017), "HFT: not so flashy anymore", June 14, available at: www.thetradenews.com/ Technology/HFT-Not-so-flashy-anymore/?p=2 (accessed June 2017).

Potters, M. and Bouchaud, J.-P. (2003), "More statistical properties of order books and price impact", Physica A: Statistical Mechanics and its Applications, Vol. 324 No. 1, pp. 133-140.

Regulation National Market System (Reg NMS) (2005), "Final rules and amendments to joint industry plans", June 9, available at: www.sec.gov/rules/final/34-51808.pdf (accessed September 2005).

SEC (2010a), Concept Release on Equity Market Structure, 75 Fed Reg. 3594, 3606 (proposed) January 21, available at: www.sec.gov/rules/concept/2010/34-61358fr.pdf

SEC (2010b), Findings Regarding the Market Events of May 6, 2010, Reports of the Staffs of the CFTC and SEC to the joint Advisory Committee on Emerging Regulatory Issues, September 30, available at: www.sec.gov/news/studies/2010/marketevents-report.pdf

US Securities and Exchange Commission (2013), "Equity market structure literature review, part I: market fragmentation", US Securities and Exchange Commission, Washington, DC, October 7, available at: www.sec.gov/marketstructure/research/fragmentation-lit-review-100713.pdf

US Securities and Exchange Commission (2014), "Equity market structure literature review, part II: high frequency trading", US Securities and Exchange Commission, Washington, DC, March 17, available at: www.sec.gov/marketstructure/research/hft_lit_review_march_2014.pdf

Vazquez, L.I. (2017), "High frequency trading: is regulation the answer?", Wake Forest Journal of Business and Intellectual Property Law, Vol. 17 No. 2, pp. 153-177, available at: http://ipjournal. law.wfu.edu/files/2017/05/17-Wake-Forest-J.-Bus.-Intell.-Prop.-L.-151.pdf

\section{Corresponding author}

Ali N. Akansu can be contacted at: Akansu@NJIT.EDU

For instructions on how to order reprints of this article, please visit our website: 